\title{
Magnetic Resonance Spectroscopy: A Promise for Detection of Metabolic Changes in the Brain of Intrauterine Growth Restriction Fetuses
}

\author{
${ }^{1}$ Vlad Zamfirescu, ${ }^{2}$ Radu Vladareanu, ${ }^{3}$ Simona Vladareanu
}

\begin{abstract}
Technical and therapeutic advances have driven the development of fetal MRI, which is likely to become an increasingly important modality in the evaluation of sonographically complex or occult anomalies of the fetal brain and body. All radiologists involved in prenatal imaging should be aware of the applications and limitations of this modality.

Magnetic resonance imaging (MRI) studies have consistently demonstrated brain structural changes on intrauterine growth restriction (IUGR). There is a need to improve MRI characterization of the anatomical patterns of brain reorganization associated with IUGR and to develop specific imaging biomarkers. Contrary to acute perinatal events, IUGR is a chronic condition that induces brain reorganization and abnormal maturation rather than gross tissue destruction. Diffusion MRI offers a promising approach to assess abnormalities in brain maturation and develop biomarkers for clinical use. The association between IUGR and short- and long-term neurodevelopmental and cognitive dysfunctions has been extensively described. Preliminary clinical results suggest that diffusion MRI could also be suitable to detect maturational changes occurring in chronic fetal conditions, including fetal cardiac defects and IUGR.
\end{abstract}

Keywords: Brain, Fetus, Intrauterine growth restriction, Magnetic resonance spectroscopy, $\mathrm{N}$-acetylaspartate.

How to cite this article: Zamfirescu V, Vladareanu R, Vladareanu S. Magnetic Resonance Spectroscopy: A Promise for Detection of Metabolic Changes in the Brain of Intrauterine Growth Restriction Fetuses. Donald School J Ultrasound Obstet Gynecol 2015;9(1):40-43.

Source of support: Nil

Conflict of interest: None

Intrauterine growth restriction (IUGR) due to placental insufficiency affects 5 to $10 \%$ of all pregnancies and induces cognitive disorders in a substantial proportion of

\footnotetext{
${ }^{1}$ Assistant Professor, ${ }^{2}$ Professor and Chairman

${ }^{3}$ Associate Professor and Head

${ }^{1,2}$ Department of Obstetrics and Gynecology, Carol Davila University of Medicine, Elias University Hospital, Bucharest Romania

${ }^{3}$ Department of Neonatology, Carol Davila University of Medicine, Elias University Hospital, Bucharest, Romania

Corresponding Author: Vlad Zamfirescu, Assistant Professor Department of Obstetrics and Gynecology, Carol Davila University of Medicine, Elias University Hospital, Baltagului 6 Bucharest, Romania, Phone: 0040744508036, e-mail: vlad zamf@yahoo.com
}

children of all newborns and is associated with a highrisk of abnormal neurodevelopment. The timing and patterns of brain reorganization underlying IUGR are poorly documented. Reduction of placental blood flow results in chronic exposure to hypoxemia and undernutrition and this has consequences on the developing brain. The association between IUGR and short- and long-term neurodevelopmental and cognitive dysfunctions has been extensively described. Additionally, magnetic resonance imaging (MRI) studies have consistently demonstrated brain structural changes on IUGR. Decreased volume in gray matter (GM) and hippocampus, and major delays in cortical development have been reported in neonates as well as reduced GM volumes and decreased fractal dimension of both GM and white matter (WM) in infants.

There is a need to improve MRI characterization of the anatomical patterns of brain reorganization associated with IUGR and to develop specific imaging biomarkers. Contrary to acute perinatal events, IUGR is a chronic condition that induces brain reorganization and abnormal maturation rather than gross tissue destruction. Consequently, it requires the use of MRI modalities allowing to identify subtle changes in brain structure. Among these, diffusion MRI offers a promising approach to assess abnormalities in brain maturation and develop biomarkers for clinical use.

Magnetic resonance spectroscopy represents an invaluable tool for the in vivo study of brain development at the chemistry level. Fetal magnetic resonance spectroscopy (MRS) is technically feasible in utero and demonstrates similar findings to those observed in neonatal populations. MRS can provide additional information to conventional T1- and T2-weighted imaging of the fetal brain. It is of particular use when subtle changes are present on conventional fetal MRI sequences, and when imaging fetuses at risk of brain injury and metabolic abnormalities. The changing anatomy of the fetal brain during maturation is also challenging. Gyration of the gray matter starts at 18 weeks' gestational age (GA); until 35 weeks, the entire primary and most of the secondary sulci are present. Up to approximately 30 weeks, the brain parenchyma differs markedly from the postnatal stages. 
MR spectroscopy has been established as a useful tool for understanding the pathophysiologic mechanisms of fetal brain injury. ${ }^{1-4}$ The intrinsic magnetic properties of the fetal brain are used to compile a spectrum by using the chemical-shift phenomenon. The spectrum contains different signal peaks based on identifiable metabolites. ${ }^{1,5}$ The composition of these metabolites may indicate abnormalities in the fetal brain metabolism, such as those that occur in developmental disorders; inborn errors of metabolism; and hypoxic encephalopathy. ${ }^{6-10}$ MR spectroscopy is a complementary adjunctive tool to morphologic imaging of the brain. ${ }^{1}$ However, the quality of the spectroscopic studies strongly depends on the homogeneity of the magnetic field and may be limited because of unrestricted fetal movement during the acquisition of the scan. Fetal sedation is recommended to avoid these motion artifacts. ${ }^{5,11,12}$ Nevertheless, on the basis of routine clinical experience, fetal sedation is usually not necessary. This may be primarily dependent on the gestational age and fetal head position. In the older and larger fetus, less movement in utero is possible. In the cephalic position, the fetal head may be more fixed in the maternal pelvis, preventing gross movement of the head. Prenatal MR spectroscopy of the brain is feasible in most unsedated fetuses, regardless of the type of spectra, fetal presentation, GA or fetal pathology. Nevertheless, approximately one-third of MR spectroscopy studies in unsedated fetuses are of limited quality.

Maternal premedication with $10 \mathrm{mg}$ diazepam (benzodiazepine) is considered to be a safe method for both mother and fetus. ${ }^{9,12}$ Brunel et $\mathrm{al}^{11}$ suggest the oral administration of flunitrazepam, a short- to intermediateacting benzodiazepine, 15 minutes to 1 hour before the examination. ${ }^{13-15}$ However, even with a single maternal dose of a benzodiazepine, the mother must be monitored after the MR imaging examination.

Intrauterine growth restriction (IUGR) is a major clinical concern that complicates up to $10 \%$ of all pregnancies. ${ }^{16}$ It is associated with an increased risk of intrauterine death, neonatal death, and preterm delivery and significant postnatal morbidity. Neonates who do survive are at increased risk of neurodevelopmental sequelae in childhood and the metabolic syndrome in later life. ${ }^{17}$ True fetal growth restriction that results from placental insufficiency is a pathologic condition that is associated with abnormal Doppler velocimetry in the umbilical artery, such as an increased pulsatility index and absent or reversed end diastolic blood flow. ${ }^{18}$ True fetal growth restriction is also associated with the phenomenon known as brain sparing, ${ }^{18}$ which is defined as an increase in blood flow to the heart and brain at the expense of other organs, such as the kidneys and gastrointestinal tract.
However, this supposedly neuroprotective mechanism is unable to compensate fully, which is evidenced by the higher incidence of cerebral palsy and neurodevelopmental sequelae in children who were growth restricted in utero. ${ }^{17}$

In the fetal brain, energy is derived primarily from glucose metabolism through the Krebs cycle and oxidative phosphorylation within mitochondria. $\mathrm{N}$-acetylaspartate (NAA) is a neuronal marker that also is localized to both mature and immature oligodendrocytes, is synthesized in neuronal mitochondria, is considered to reflect mitochondrial function..$^{19}$ Proton MRS is a technique that asses noninvasively the chemical composition of in vivo tissue and investigates differences in brain levels of NAA between appropriately grown and growthrestricted human fetuses. In the healthy neonatal brain, MRS reveal four main groups of metabolites: NAA, choline, creatine and inositol (Ino) (Graph 1). Normative values for the levels of the fetal brain metabolites, Ino, $\mathrm{Cho}, \mathrm{Cr}$, and NA, and their ratios are available and can be used as references to examine changes in MRS spectra due to pathological conditions of the fetal brain, for example, neuronal damage due to hypoxia.

Reduced cerebral levels of NAA that are evidenced by a reduction in NAA:choline (Cho) and NAA: creatine (Cr) ratios will be present in IUGR fetuses, which may be indicative of abnormal mitochondrial metabolism and may predispose these infants to the increased incidence of perinatal brain injury and later neurodevelopmental impairments (Graph 2).

Whereas magnetic resonance spectroscopy has received wide attention in pediatric and adult settings, only a few studies were performed on the human fetal brain. They revealed changes occurring throughout gestation in the levels of the main metabolites detected by proton magnetic resonance spectroscopy ( $\mathrm{N}$-acetylaspartate, choline, myoinositol, creatine and glutamate),

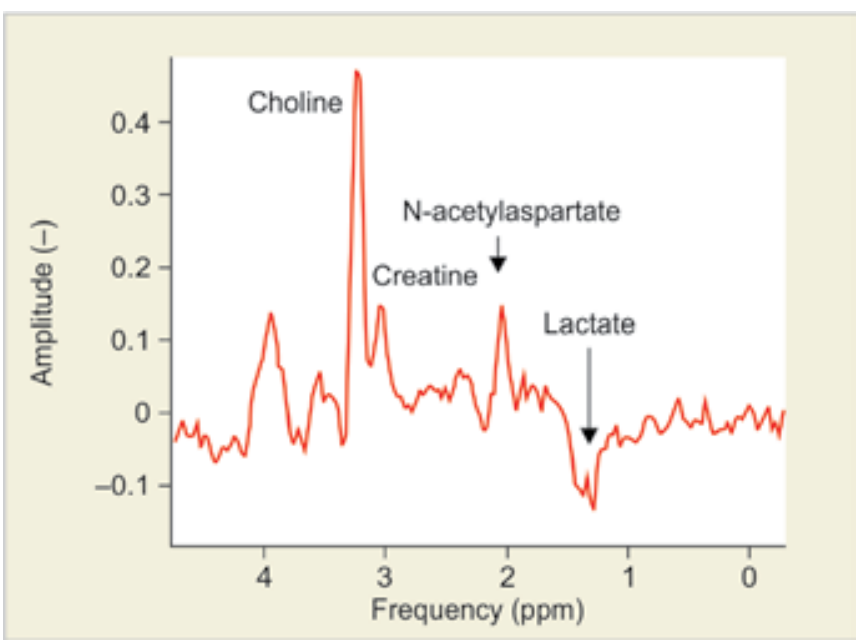

Graph 1: Twenty-four weeks normal fetus 


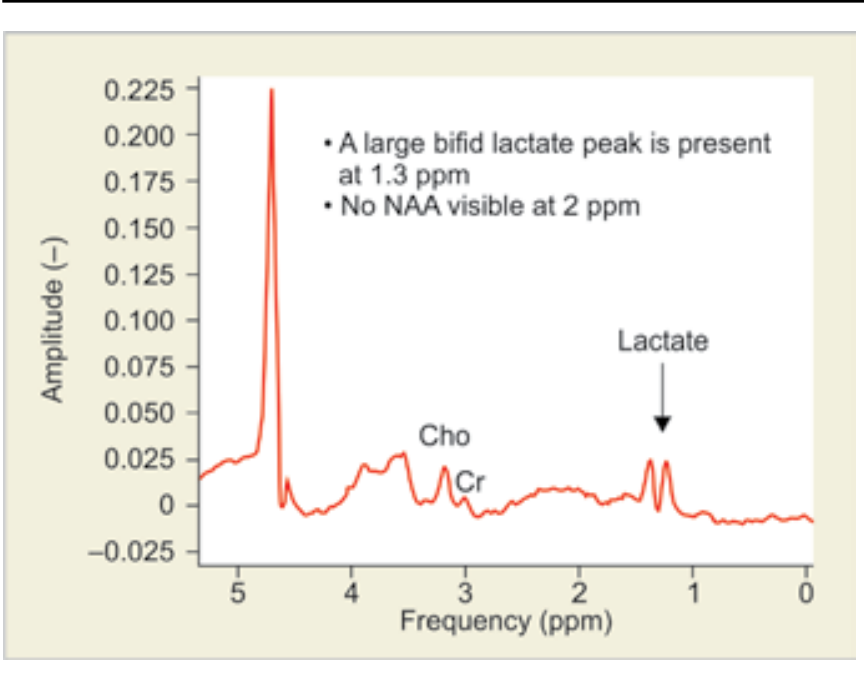

Graph 2: Twenty-four weeks IUGR fetus

providing a reference for the normal metabolic brain development. Throughout the third trimester of gestation, $\mathrm{N}$-acetylaspartate gradually increases, whereas choline undergoes a slow reduction during the process of myelination.

Cerebral redistribution is associated with altered brain metabolism that is evidenced by a reduction in NAA:Cho and NAA:Cr ratios. We compared the levels of choline, NAA, lactate, creatine detected in the brains of normal fetuses and of those with IUGR and we observed that in the brains of the IUGR fetuses the levels of NAA is undetectable and lactate is increased while the choline and creatine are lower.

Metabolite levels within the human fetal brain have been shown to change with increasing gestational age during the third trimester. This finding is thought to reflect maturation, in agreement with MRS performed on preterm neonates. At 22 weeks of gestation the MRS is characterized by two prominent resonances assigned to Myo-ino and Cho. The Myo-ino resonance dominates the spectrum from 22 to 28 weeks of gestation and is significantly reduced with progressing gestational ages in fetuses. Choline is also prominent on the MRS spectrum from 22 to 28 weeks. Cho is involved in synthesis of acetylcholine and membrane phospholipids and is taken up by both glia and neurons. Cho and the Cho:Cr ratio significantly decrease with increasing gestational age. NAA also appears in the spectrum as early as 22 weeks although the peak intensity is weak and is thought to be related to the development of dendrites and synapses as well as proliferation and differentiation of oligodendrocytes. NA signal increases significantly with progressing gestational age. Ino shows no significant change in relation to fetal gestational age. Increasing cerebral tissue levels of NA and $\mathrm{Cr}$ and decreasing levels of $\mathrm{Cho}$ and Ino are observed with the development of the fetal brain. At 34 weeks of gestation, the spectrum of metabolites is similar to that of the neonate. Recently, lactate was shown on MRS studies in IUGR fetuses and in patients with gastroschisis. However, as lactate may be part of the normal metabolic spectrum in premature newborns, the pathological significance of a lactate peak found in the fetal brain remains unclear. In IUGR fetuses with normal morphology, MRS of the fetal brain shows lactate and a low NAA:Cho index, metabolic markers of starvation/ hypoxia. This pattern is consistent with the metabolic changes seen in hypoxic ischemic injury in neonates. A recent study additionally shows a significant increase in Ino:Cho ratio and significantly higher apparent diffusion coefficient values in the pyramidal tract of small for gestational age fetuses compared with age-matched normal fetuses.

Another field for investigation involves the possibility of early detection of glutamate levels in fetuses at risk for hypoxic-ischemic encephalopathy, because the role of glutamate excitotoxicity in this context is well-established. Because metabolic modifications may precede functional or morphologic changes in the central nervous system, magnetic resonance spectroscopy may likely serve as a powerful, noninvasive tool for the early diagnosis and prognosis of different pathologic conditions.

\section{CONCLUSION}

Fetal MRS is a challenging technique. MRI has the potential to become an important tool in studying normal and abnormal fetal brain development. This relevant not only to conditions leading to serious brain damage (cerebral palsy) but also to conditions such as IUGR leading to minor damage. It may also be relevant for understanding adult brain function. In conclusion, fetal MR-methods may be used to receive functional and metabolic information of the fetus and placenta respectively.

In addition to morphology these methods give further insight into normal and pathological prenatal development. Using fetal MRI in addition to prenatal ultrasound, morphological, metabolic, and functional assessment of the fetus can be achieved. Further investigations are needed to prove the diagnostic potential of these new imaging techniques.

\section{REFERENCES}

1. Pugash D, Krssak M, Kulemann V, et al. Magnetic resonance spectroscopy of the fetal brain. Prenat Diagn 2009;29: 434-441.

2. Huppi PS, Amato M. Advanced magnetic resonance imaging techniques in perinatal brain injury. Biol Neonate 2001;80: 7-14.

3. Toft PB, Leth H, Lou HC, et al. Metabolite concentrations in the developing brain estimated with proton MR spectroscopy. J Magn Reson Imaging 1994;4:674-680. 
4. Cady EB, Amess P, Penrice J, et al. Early cerebral-metabolite quantification in perinatal hypoxic-ischemic encephalopathy by proton and phosphorus magnetic resonance spectroscopy. Magn Reson Imaging 1997;15:605-611.

5. Girard N, Gouny SC, Viola A, et al. Assessment of normal fetal brain maturation in utero by proton magnetic resonance spectroscopy. Magn Reson Med 2006;56:768-775.

6. Kok RD, Steegers-Theunissen RP, Eskes TK, et al. Decreased relative brain tissue levels of inositol in fetal hydrocephalus. Am J Obstet Gynecol 2003;188:978-980.

7. Azpurua H, Alvarado A, Mayobre F, et al. Metabolic assessment of the brain using proton magnetic resonance spectroscopy in a growth-restricted human fetus: case report. Am J Perinatol 2008;25:305-309.

8. Astley SJ, Richards T, Aylward EH, et al. Magnetic resonance spectroscopy outcomes from a comprehensive magnetic resonance study of children with fetal alcohol spectrum disorders. Magn Reson Imaging 2009;27:760-778.

9. Roelants-van Rijn AM, Groenendaal F, Stoutenbeek P, et al. Lactate in the foetal brain: detection and implications. Acta Paediatr 2004;93:937-940.

10. Roelants-Van Rijn AM, van der Grond J, de Vries LS, et al. Value of 1H-MRS using different echo times in neonates with cerebral hypoxia- ischemia. Pediatr Res 2001;49:356-362.

11. Brunel H, Girard N, Confort-Gouny S, et al. Fetal brain injury. J Neuroradiol 2004;31:123-137.
12. Gerards FA, Stoutenbeek P, Gooskens RH, et al. Diagnostic value of prenatal MRI in fetus with intracranial anomalies diagnosed by ultrasonography [in Dutch]. Ned Tijdschr Geneeskd 2001;145:179-184.

13. Kanto J, Erkkola R, Kangas L, et al. Placental transfer of flunitrazepam following intramuscular administration during labour. Br J Clin Pharmacol 1987;23:491-494.

14. Oelschläger H. Chemical and pharmacologic aspects of benzodiazepines [in German]. Schweiz Rundsch Med Prax 1989:78:766-772.

15. Kanto JH. Use of benzodiazepines during pregnancy, labour and lactation, with particular reference to pharmacokinetic considerations. Drugs 1982:23:354-380.

16. American College of Obstetricians and Gynecologists, Intrauterine growth restriction. ACOG practice bulletin no. 12. Washington, DC: The College; 2000.

17. Pallotto EK, Kilbride HW. Perinatal outcome and later implications of intrauterine growth restriction. Clin Obstet Gynecol 2006;49:257-269.

18. Mari G, Hanif F. Intrauterine growth restriction: how to manage and when to deliver. Clin Obstet Gynecol 2007; 50:497-509.

19. Vielhaber S, Niessen HG, Debska-Vielhaber G, et al. Subfieldspecific loss of hippocampal $\mathrm{N}$-acetyl aspartate in temporal lobe epilepsy. Epilepsia 2008;49:40-50. 